Abstract PTU-181 Table

\begin{tabular}{|c|c|c|c|c|c|c|}
\hline Age & Patients & $\begin{array}{l}\text { Normal duodenum } \\
\text { (non-targeted biopsy) }\end{array}$ & $\begin{array}{l}\text { Non- targeted biopsy, } \\
\text { known CD }\end{array}$ & $\begin{array}{l}\text { Non- targeted biopsy for } \\
\text { other indication }\end{array}$ & $\begin{array}{l}\text { Non-targeted biopsy to } \\
\text { exclude CD }\end{array}$ & $\begin{array}{l}\text { Non-targeted biopsy, giving } \\
\text { new CD diagnosis }\end{array}$ \\
\hline$\leq 54$ & 88 & 73 & 6 & 4 & 63 & 5 \\
\hline$\geq 75$ & 81 & 65 & 0 & 0 & 65 & 0 \\
\hline Total & 297 & 237 & 12 & 7 & 218 & 8 \\
\hline
\end{tabular}

significant pathology would be missed as a result. We therefore support the approach of tTG testing prior to endoscopy, to reduce unnecessary biopsies. No new diagnoses of CD were made in patients $\geq 75$ years in our study.

Disclosure of Interest None Declared.

\section{PTU-182 ABDOMINAL PAIN DISTINGUISHES IDIOPATHIC BILE ACID MALABSORPTION (BAM) FROM DIARRHOEA- PREDOMINANT IRRITABLE BOWEL SYNDROME (IBS-D)}

\author{
doi:10.1136/gutjnl-2013-304907.272
}

1."B Kok, 'A Mistry, 'R Malhotra, 'S Townsend, ' ${ }^{1}$ A Milestone, 'M Hall. 'Department of Gastroenterology, Hereford County Hospital, Hereford, UK

Introduction Current BSG guidelines for chronic diarrhoea recommend that patients aged $<45$ with diarrhoea suggestive of functional disease (ie $>3$ months duration, no weight loss, no nocturnal symptoms, intermittent symptoms) and normal basic investigations, do not require further investigation and have IBS ${ }^{1}$. This potentially disadvantages a population who might in fact suffer with idiopathic BAM. NICE guidelines however feature abdominal pain as one of the key symptoms for diagnosing IBS ${ }^{2}$. In this observational study we aim to assess whether abdominal pain can distinguish idiopathic BAM from IBS-D.

Methods All patients who underwent SeHCAT scan over a 30 month period January 2009-June 2012 were identified. Patient records and blood results/radiological imaging/endoscopy procedures performed prior to SeHCAT scan were reviewed.

Results A total of 112 patients were identified. 4 patients were excluded due to the unavailability of patient records. 53 patients $(49 \%)$ had abnormal bile acid retention on SeHCAT (defined by $<$ $8 \%$ retention). Of these 53 patients, $72 \%$ were female $(n=38)$ with a median age of 52 years (range 26-80) and average stool frequency of 7 times/day. 27 of the 53 patients (51\%) had no known risk factors for BAM and the other 26 patients had risk factors (eg terminal ileal disease/ileal resection/post-cholecystectomy). Of the 55 patients classified as having normal bile acid retention ( $>8 \%$ retention), 39 of the 55 (71\%) had no known risk factors for BAM.

Of the 39 patients who had suspected idiopathic BAM but normal SeHCAT, excluding 12 patients where there was no clear mention of abdominal pain in the notes, $78 \%$ had abdominal pain as a prominent symptom $(21 / 27)$ and $22 \%$ had no pain. Of the 27 patients who had suspected idiopathic BAM and abnormal SeHCAT, excluding 7 patients where there was no mention of abdominal pain in the notes, only $40 \%$ had abdominal pain $(8 / 20)$ whereas $60 \%$ had no pain (Chi-squared, $\mathrm{p}=0.008$ ).

20 of the 53 patients with BAM were aged $<45$ years old. 6 of these 20 had known risk factors for Type I BAM due to history of terminal ileal disease but the remaining 14 patients had no risk factors, fit criteria for functional disease, and could easily have been labelled as IBS-D.

Conclusion Our results highlight that a significant proportion of patients with chronic diarrhoea suffer from BAM. Younger patients cannot be assumed to have 'functional' symptoms and though the population is small, it does seem that idiopathic BAM patients might be distinguishable from IBS-D patients in that they usually do not experience abdominal pain.

Disclosure of Interest None Declared.

\section{REFERENCES}

1. Thomas PD et al., Gut 2003; 52 (Suppl V): v1-v15 - Guidelines for the investigation of chronic diarrhoea, 2nd edition

2. NICE guidelines on Irritable Bowel Syndrome (CG61) 2008

\section{PTU-183 SEHCAT - CAST A WIDER NET}

doi:10.1136/gutjnl-2013-304907.273

1."B Kok, 'A Mistry, 'R Malhotra, 'J Burdsall, ' $\mathrm{M}$ Hall, 'R Ransford, 'A Milestone. 'Department of Gastroenterology, Hereford County Hospital, Hereford, UK

Introduction Bile acid malabsorption (BAM) is a common cause for chronic diarrhoea in patients with risk factors. The diagnosis is usually confirmed by SeHCAT scan demonstrating lack of retention of radiolabelled bile acid. Treatment with bile acid sequestrants may improve diarrhoea symptoms. We report our experience of using SeHCAT in the investigation of patients with chronic diarrhoea and patient response to therapy.

Methods Over a 30 month period January 2009- June 2012, all patients who underwent a SeHCAT scan were identified. Patient records and blood results/radiological imaging/endoscopy procedures performed prior to SeHCAT were reviewed. An abnormal SeHCAT result was defined by bile acid retention $<8 \%$. Equivocal results of $8-15 \%$ retention were regarded as normal.

Results 112 patients underwent a SeHCAT scan during this period. 4 patients were excluded due to unavailability of patient records. 53 patients $(49 \%)$ had abnormal SeHCAT retention. In the remaining 55 patients bile acid retention ranged from $8.1 \%$ to $76.8 \%$. BAM was diagnosed in 8 out of 17 patients with a possible Type I abnormality (terminal ileal disease/resection or previous pelvic radiotherapy), 27 out of 66 patients with a possible Type II abnormality (idiopathic) and 18 out of 25 patients with a possible Type III abnormality (post-cholecystectomy). Additional patient demographics are represented in Table 1 .

The incidence for BAM was highest in the cohort of patients who were post-cholecystectomy, with $71 \%$ of all suspected Type III BAM patients having abnormal SeHCAT. However, in terms of absolute numbers, idiopathic BAM formed the highest proportion for abnormal SeHCAT.

Interestingly, a wide age variation was experienced across the three groups with proven BAM. The age range in Type I was 36-71 years, in Type II 37-74 years and in Type III 26-80 years.

$57 \%$ of patients with proven BAM had documented improvement in their diarrhoea frequency and consistency on treatment with a bile acid sequestrant (eg colestyramine). Average reduction of stool frequency was from 7 times/day to 3 times/day.

Conclusion These results show that BAM is a relatively common problem. It occurs for a variety of reasons and should be thought of earlier in the investigative algorithm, independent of age. In our cohort, a history of post-cholecystectomy chronic diarrhoea was the most likely risk factor associated with BAM, hence this group 